\title{
BIODEGRADAÇÃO DE BIODIESEL POR CONSÓRCIO DE MICRO-ORGANISMOS DEGRADADORES DE HIDROCARBONETO
}

\author{
M. F. A. da ROCHA ${ }^{1}$, O. M. MARQUES ${ }^{1}$ e S. M. SARMENTO ${ }^{1}$ \\ ${ }^{1}$ Universidade Federal de Pernambuco - Departamento de Engenharia Química \\ E-mail para contato: smsarmento@gmail.com
}

\begin{abstract}
RESUMO - O processo de biodegradação de um composto orgânico (biocombustíveis) permite a atuação de micro-organismos na recuperação de áreas contaminadas por este, e que pode ser acompanhado pela evolução dinâmica do $\mathrm{CO}_{2}$, entre outros métodos. Neste trabalho, foi avaliada a degradação em meio aquoso do biodiesel de algodão (B100-A) através da concentração de biomassa microbiana, teor de éster metílico de ácidos graxos (EMAG), pH, DQO e $\mathrm{DBO}_{5}$. Usou-se o consórcio de micro-organismos Pseudomonas aeruginosa e Saccharomyces cereviseae não aclimatados e pré-selecionados pela técnica do indicador redox 2,6-diclorofenol-indofenol dihidratado (DCPIP). Resultados deste estudo mostraram que : a) O B100-A apresentava em sua composição $27,2 \%$ de EMAG saturados (C16:0 e C18:0) e 72,8 \% insaturados (C18:1 e C18:2); b) O pH inicial $(6,0)$ mostrou maior redução $(3,1)$ ao $35^{\mathrm{a}}$ dia de processo resultando a redução de células viáveis $\left(10^{6}\right.$ a $10^{4}$ UFC.cm $\left.{ }^{-3}\right)$ entre 14 a 35 dias; c) O EMAG C18:2 decompôs-se em C16:0 e C18:0. Do $7^{\mathrm{a}}$ ao $35^{\mathrm{a}}$ dia transformaram-se entre si os EMAG C16:0, C18:0 e C18:1. Os produtos da degradação destes mostraram que a $\mathrm{DBO}_{5}$ aumentou $(522,7 \%)$ do 0 a $14^{\mathrm{a}}$ dia e $(6,6 \%)$ do $14^{\mathrm{a}}$ ao $28^{\mathrm{a}}$ dia que indicou redução da biodegradabilidade do B100-A pela redução da oxidação destes verificada pela DQO. Conclui-se que o B100-A sofreu degradação primária em presença do consórcio microbiano associada a concentração de biomassa relacionada à variação de $\mathrm{pH}$ da fase aquosa sem controle do $\mathrm{pH}$.
\end{abstract}

\section{INTRODUÇÃO}

O Biodiesel (B100) é um combustível alternativo, produzido a partir de recursos renováveis provenientes da biomassa, o que diminui a dependência do petróleo e como tal, tem recebido intensa atenção devido o rápido crescimento do preço deste insumo e a preocupação com o meio ambiente. O B100 apresenta alto potencial de biodegradabilidade, pois é composto por ésteres alquílicos de ácidos orgânicos, EAAG (Ferrari et al., 2005). É totalmente solúvel no diesel (B0) cujas misturas têm sido formuladas (Misturas B0/B100) com proporção de B100 que podem variar de 1 a $99 \%$ (v/v). Especificamente no Brasil, o B100 tem sido utilizado como aditivo ao B0 com percentual de $5 \%$ (v/v) desde 2010 (Kardec, 2011). Dados estatísticos mostram que, no Brasil, atividades como carga e descarga de petróleo e derivados, operações de lavagem de tanques de navios, tratamentos de óleo entre outras, contribuem com $90 \%$ da poluição por hidrocarbonetos de petróleo. Acidentes com transporte contribuem com apenas 5 
a $10 \%$ da poluição, porém, sua ocorrência é muito mais danosa ao meio ambiente, caso não seja controlada rapidamente (Vital, 1992 ; Ururahy, 1998). Portanto, com a comercialização do B0, contendo B100 como aditivo, há necessidade de se estudar o possível impacto deste no meio ambiente aquático (Rodrigues, 2009).

Poucos estudos têm sido realizados sobre a biodegradação do B100 em ambiente aquático e como este impactaria a degradação do B0 nesse ambiente. De acordo com (Zhang et al., 1998), o B100 pode promover e acelerar a biodegradação aeróbia do B0 em misturas aquosas contendo nutrientes e biomassa por meio do processo biológico conhecido como cometabolismo. Geralmente, na biodegradação de compostos orgânicos, a atividade microbiana é o mecanismo predominante (Kemp, 2003) como alternativa viável na descontaminação de locais impactados que consiste na utilização de um ou mais consórcios microbianos, indígenas ou não, para a degradação de contaminantes orgânicos (Pereira e Lemos, 2006) e a obtenção de energia podendo ser averiguada preliminarmente usando indicadores desta, tais como, a produção de dióxido de carbono, consumo de oxigênio, evolução dinâmica do $\mathrm{pH}$, $\mathrm{DQO}$ e $\mathrm{DBO}_{5}$ no meio (Zhang et al., 1998). Os micro-organismos quebram as moléculas do composto orgânico poluente em outras com estruturas moleculares mais simples (processo de biodegradação), que podem seguir quebrando-se em outras mais simples até atingirem, por fim, o nível químico (mineralização biótica).

Rocha et al. (2011) estudaram a biodegradação do B100-A em sistema aeróbio em presença do consórcio constituído por culturas puras ( $P$. aeruginosa e $S$. cereviseae) préselecionados pela técnica do indicador redox DCPIP e não-aclimatadas cujo desenvolvimento foi acompanhado através de monitoramento, por período total de 35 dias, dos seguintes parâmetros de processo: concentração dos EMAG, pH, $\mathrm{DBO}_{5}$ e DQO.

Verificou-se que o B100-A sofreu degradação primária cujo EMAG C18:2 se decompôs totalmente nos 7 primeiros dias do processo, no entanto os C16:0, C18:0 e C18:1 decompuseram em determinados períodos do processo, em outros, porém, são produtos da decomposição de alguns desses EMAG cuja produção ocorreu com intensidade máxima nos 7 primeiros dias do processo. Esses EMAG passaram a decomporem-se no intervalo de tempo de 7 a 21 dias de processo, em seguida, oscilaram entre produto e reagente indicando que transformaram se entre si. O processo de degradação do B100-A foi acompanhado através do pH como sensor de ocorrência de biodegradação do sistema, cuja população de biomassa apresentou fase de adaptação (0 a 7 dias), crescimento (14 a 21 dias) e morte celular (7 a 14, 21 a 35 dias). Os produtos da degradação dos EMAG ao longo do processo mostraram ser menos biodegradáveis que seus precursores, gerando maior demanda de oxigênio dissolvido da fase aquosa do B100-A para sua biodegradação aeróbia verificado pela avaliação da $\mathrm{DBO}_{5}$ e DQO principalmente após o $7^{\circ}$ dia de processo. O objetivo deste trabalho foi verificar a biodegradação do B100-A da fase líquida através da concentração de biomassa microbiana, teor de éster metílico de ácidos graxos (EMAG), pH, DQO e $\mathrm{DBO}_{5}$ quando no processo houver ausência do controle de $\mathrm{pH}$. 


\section{MATERIAS E MÉTODOS}

\subsection{Materiais}

Biodiesel de algodão: O B100-A não aditivado foi cedido pelo Centro de Tecnologias Estratégicas do Nordeste- CETENE, cujas propriedades físico-químicas são mostradas na Tabela 1. Este combustível em estudo foi esterilizado por meio de filtração a vácuo em membrana Millipore (Marca Gelman Sciences 0,22 $\mu \mathrm{m}$ ) e acondicionado em frasco âmbar estéril.

Tabela 1- Propriedades físico-químicas do biodiesel de algodão (Fonte: CETENE)

\begin{tabular}{ccccc}
\hline Combustível & $\begin{array}{c}\mathrm{ET}^{*} \\
\left(\mathrm{mg} \cdot \mathrm{Kg}^{-1}\right)\end{array}$ & $\begin{array}{c}\rho 20^{\circ} \mathrm{C} \\
\left(\mathrm{Kg}^{-3}\right)\end{array}$ & $\begin{array}{c}\mu 40^{\circ} \mathrm{C} \\
(\mathrm{cSt})\end{array}$ & $\begin{array}{c}\mathrm{PF}^{* *} \\
\left({ }^{\circ} \mathrm{C}\right)\end{array}$ \\
\hline B100-A & 0 & 883,1 & 4,8 & 152 \\
\hline
\end{tabular}

*ET (Enxofre Total), **PF (Ponto de Fulgor), $\rho$ (massa especifica), $\mu$ (viscosidade absoluta).

Micro-organismos: As culturas microbianas puras que constituíram o consórcio microbiano foram a Pseudomonas aeruginosa (ATCC 27853- DAUFPE 416) e Saccharomyces cereviseae (ATCC 5107- DEQUFPE) cujos meios de cultivo foram Agar Mueller-Hinton (HIMEDIA) e Agar de Sabouraud (Merck), respectivamente. Estas culturas foram mantidas acondicionadas sob refrigeração $\left(5^{\circ} \mathrm{C}\right)$ e repicadas a cada dois meses. Usou-se no preparo do inoculo o meio de cultura mínimo (MCM): $0,5 \% \mathrm{NaCl} ; 0,1 \% \mathrm{~K}_{2} \mathrm{HPO}_{4} ; 0,1 \% \mathrm{KH}_{2} \mathrm{PO}_{4} ; 0,1 \%\left(\mathrm{NH}_{4}\right)_{2} \mathrm{SO}_{4}$; $0,02 \% \mathrm{MgSO}_{4} .7 \mathrm{H}_{2} \mathrm{O} ; 0,3 \% \mathrm{KNO}_{3} ;$ acrescido de glicose.

\subsection{Métodos}

Meio de crescimento e inoculo: Inoculos individuais $\left(10 \mathrm{~cm}^{3}\right)$ das espécies microbianas que constituíram o consórcio microbiano em estudo foram preparados usando-se o MCM, sendo mantidos a $30{ }^{\circ} \mathrm{C}$ por $72 \mathrm{~h}$. Frascos Erlenmeyer $\left(1000 \mathrm{~cm}^{3}\right)$ contendo $25 \mathrm{~cm}^{3}$ dessas culturas (concentração em UFC padronizada em $10^{8} \mathrm{UFC} . \mathrm{cm}^{-3}$ na proporção de bactéria: levedura: 1:1) em $100 \mathrm{~cm}^{3}$ de MCM mantidos sob agitação orbital $(120 \mathrm{rpm})$, a $30{ }^{\circ} \mathrm{C}$ por $72 \mathrm{~h}$ de cultivo em Câmara incubadora (Marca Marconi, modelo MA-420).

Sistema experimental: O ensaio biótico, em duplicata, foi conduzido em microcosmos formado por frascos Erlenmeyer $\left(2000 \mathrm{~cm}^{3}\right)$, contendo meio MCM sem glicose $\left(700 \mathrm{~cm}^{3}\right)$, suspensão do inoculo $\left(250 \mathrm{~cm}^{3}\right)$ e B100-A $\left(50 \mathrm{~cm}^{3}\right)$ submetidos à agitação orbital $(120 \mathrm{rpm})$ a $30{ }^{\circ} \mathrm{C} \mathrm{em}$ câmara incubadora (Marca Marconi, modelo MA-420) por período total de 35 dias.

Ensaios de biodegradação e determinações analíticas: Realizaram-se amostragens a cada 7 dias, incluindo-se o dia 0 de processo monitorado através do acompanhamento das evoluções da 
concentração de células viáveis (X), concentração de EMAG, $\mathrm{DBO}_{5}, \mathrm{DQO}$ e pH da fase liquida. $1 \mathrm{~cm}^{3}$ da amostra foi utilizado para avaliar-se a concentração de células viáveis (X), pelo método de contagem em placas (pour plate) usando-se o meio de cultura "Plate Count Agar" (PCA) (Merck). $\mathrm{O}$ restante do volume da amostra foi submetido à filtração a vácuo em membrana Millipore (Marca Gelman Sciences 0,22 $\mu \mathrm{m}$ ) para separar a biomassa microbiana do líquido metabólico cujo sobrenadante foi usado nas determinações do $\mathrm{pH}$ (sensor de $\mathrm{pH}$ de bancada, Marca MARCONI, modelo PA 200/PA 200P), DBO (Incubadora DBO, Marca Quimis) e DQO (digestor de DQO, Marca Merck, modelo Q- 315M26), utilizando-se o Standard Methods 5210B e 5220C, respectivamente. A concentração dos EMAG dissolvidos na fase aquosa foi obtida, usando-se parte da solução aquosa (fase polar) extraída com hexano, por cromatografia gasosa (Cromatógrafo Marca CG, modelo Master). Utilizou-se coluna cromatográfica capilar Marca Carbowax (dimensão: $30 \mathrm{~m}$ de comprimento, $5,3 \times 10^{-4} \mathrm{~m}$ de diâmetro interno e espessura da fase estacionária de $1 \times 10^{-9} \mathrm{~m}$ ), respectivamente e detector DIC e gás de arraste, hidrogênio com $99 \%$ de pureza (White Martins) com vazão de $8,3 \times 10^{-8} \mathrm{~m}^{3} / \mathrm{s}$. Os resultados obtidos apresentaram erros experimentais máximos de $1 \%$.

\section{RESULTADOS E DISCUSSÃO}

$\mathrm{Na}$ Tabela 2 consta a composição do B100-A que apresenta uma mistura de EMAG saturados $(27,2 \%)$ e insaturados $(72,8 \%)$.

Tabela 2- Perfil de EMAG do B100-A

\begin{tabular}{cccccccc}
\hline EMAG & C14:0 & C16:0 & C18:0 & C18:1 & C18:2 & C18:3 & $\begin{array}{c}\text { Ésteres } \\
\text { totais }\end{array}$ \\
\hline Teor $(\%)$ & 0,8 & 23,5 & 3 & 15,6 & 56,6 & 0,6 & 100,1 \\
\hline
\end{tabular}

As Figuras 1a e 1b apresentam concentração normalizada dos EMAG, pH e biomassa microbiana, respectivamente. Figuras $2 \mathrm{a}$ e $2 \mathrm{~b}$ mostram resultados da $\mathrm{DBO}_{5}$ e DQO ao longo do tempo de processo, respectivamente. 


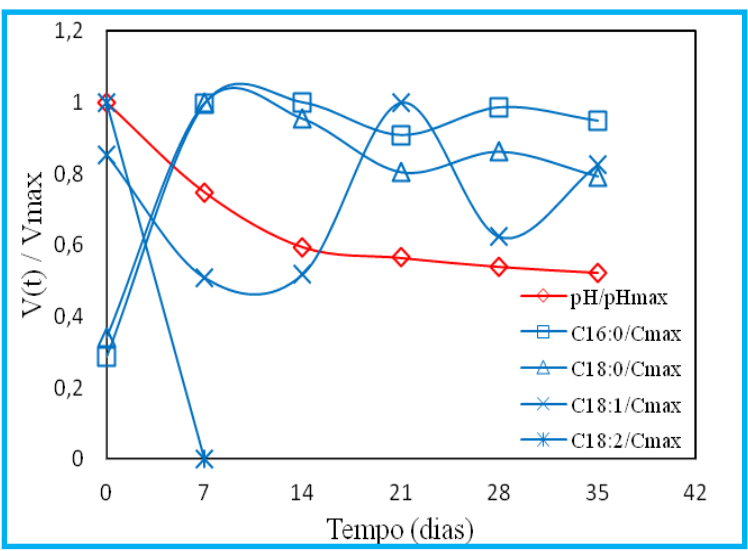

(a)

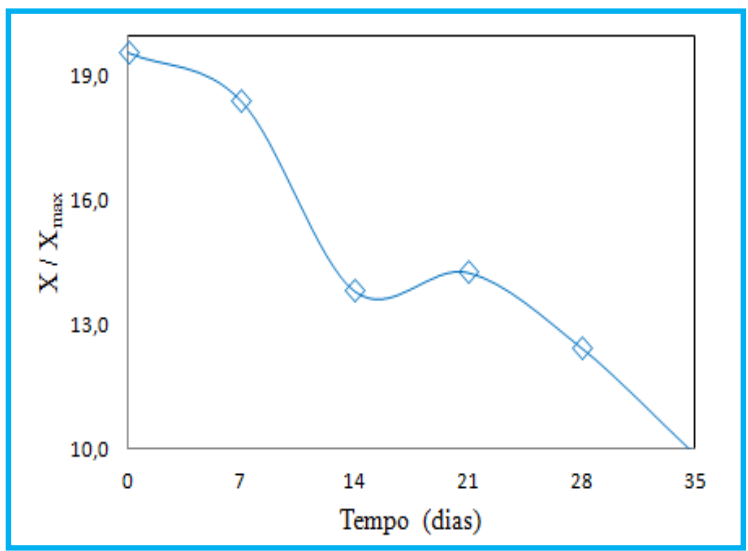

(b)

Figura 1- Evolução dinâmica de parâmetros de processo normalizados: (a) concentração de EMAG e pH; (b) concentração de biomassa microbiana. [V(t): variável, $\mathrm{V}_{\text {máx}}$ : valor máximo da variável].

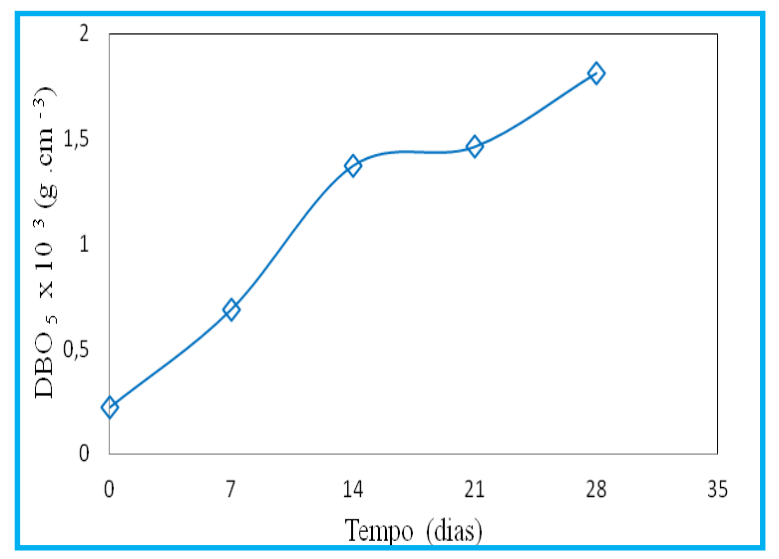

(a)

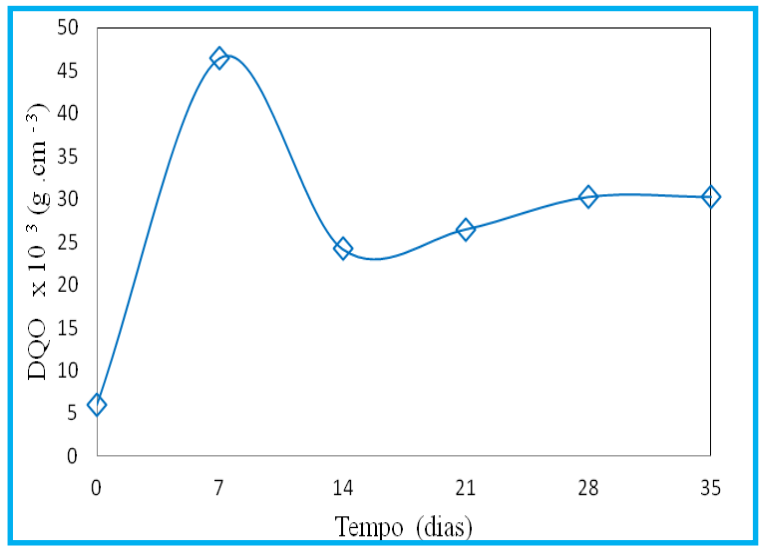

(b)

Figura 2- Evolução dinâmica de parâmetros do processo normalizados: (a) $\mathrm{DBO}_{5}$; (b) DQO.

Nas Figuras 1 e 2 pode-se verificar a variação da concentração normalizada da mistura reagente, da concentração de biomassa, $\mathrm{DBO}_{5}$ e DQO comparada com a variação do $\mathrm{pH}$ durante os cinco períodos ( 0 a 35 dias) do processo de degradação do B100-A em meio aquoso sob a ação do consórcio (P.aeruginosa e S. cerevisiae). Resultados mostraram que : a) Período 1 (0 a 7 dias) - o EMAG C18:1 decompôs-se parcialmente no EMAG C16:0, na proporção de 7,7 \% de seu teor de massa original $(15,6 \%)$. O C18:2 foi totalmente decomposto em ésteres mais estáveis, sendo eles os C16:0 e C18:0, nas proporções de 62,0 \% e 38,0 \% , respectivamente de seu teor em massa original (56,7\%). Observou-se aumento da DQO até o $7^{\circ}$ dia e pico máximo no $7^{\circ}$ dia do processo indicando neste período elevada carga orgânica enquanto que a $\mathrm{DBO}_{5}$ apresentou discreto aumento gerando demanda de oxigênio dissolvido para o processo de 
degradação. $\mathrm{O}$ pH inicial $(6,0)$ foi reduzido a 4,49 indicando a decomposição do B100 devido à formação de ácidos graxos livres de EMAG de cadeias menores que seus precursores no entanto, a população de células viáveis apresentou fase de adaptação ao meio (ordem de grandeza de $10^{8}$ UFC. $\mathrm{cm}^{-3}$ ).

b) Período 2 (7 a 14 dias) - perfis obtidos para os C16:0, C18:0 e C18:1 indicaram que o C18:0 (8,30 \%) decompôs-se parcialmente no C16:0 e C18:1, no entanto, concentrações do C16:0 (82,20 \%) e C18:1 (9,50 \%) aumentaram tornando-se produtos desta decomposição. A biodegradação da matéria orgânica apresentou aumento mais intenso até o $14^{\circ}$ dia $(522,7 \%)$ de processo gerando maior demanda de oxigênio dissolvido para sua biodegradação aeróbia, no entanto, a DQO apresentou redução da carga orgânica. Houve aumento elevado da acidez do meio aquoso $(6,00$ a 3,57) que corresponde a 49,00 \% resultando na redução de células viáveis (ordem de grandeza de $10^{8}$ UFC. $\mathrm{cm}^{-3}$ a $10^{6} \mathrm{UFC}_{\mathrm{cm}} \mathrm{cm}^{-3}$ ) responsáveis pela degradação dos contaminantes (Corseuil et al., 2003).

c) Período 3 (14 a 21 dias) - os C16:0, C18:0, C18:1 continuam fazendo parte significante da composição da mistura reagente no entanto, mantém oscilação em sua composição cujos EMAG C16:0 (74,70 \%) e C18:0 (7,00 \%) decompuseram-se parcialmente no C18:1, tornandoo produto neste período do sistema. A variação do $\mathrm{pH}$ foi pequena $(3,57$ a 3,39$)$ do $14^{\circ}$ a $21^{\circ}$ dia de processo. Os produtos da degradação neste período mostraram ser menos biodegradáveis que seus precursores cuja $\mathrm{DBO}_{5}$ mostrou aumento de $(106,6 \%)$ gerando menor demanda de oxigênio dissolvido da fase aquosa do B100-A comparada ao período anterior enquanto que, a DQO apresentou discreto aumento (57\%).

d) Período 4 (21 a 28 dias) - o EMAG C18:1 se decompôs parcialmente nos ésteres C16:0 e C18:0, considerando-se estes como produtos da decomposição destes EMAG cuja biodegradação da matéria orgânica e DQO apresentaram aumento discreto da carga orgânica devido seus produtos serem menos biodegradáveis que seus precursores quando comparado ao período 3 (124\% e 114,3\%), respectivamente. $\mathrm{O} \mathrm{pH}$ de 3,39 passou a 3,24 reduzindo a ordem de grandeza de $10^{6}$ a $10^{5}$ UFC.cm ${ }^{-3}$.

e) Período 5 (28 a 35 dias) - a concentração dos EMAG C16:0 (78,00 \%), C18:0 (6,90 $\%)$ e C18:1 (15,10 \%) continuaram a variar, entre si, havendo uma espécie de oscilação na composição desses, que ora decompõem-se, ora são produtos passando o C16:0 e o C18:0 a decompor-se no EMAG C18:1. O pH ao final dos 35 dias apresentou valor de 3,14. A decomposição dos C18:2 e C18:1 no período 1 ( 0 a 7 dias) deve-se ao fato destes apresentarem ligações insaturadas em sua molécula uma vez que, a biodegradabilidade de compostos orgânicos é indiretamente proporcional ao número de átomos de carbono e de ramificações presentes neste (Morrison e Boyd,1992). Portanto, o B100-A apresenta alta susceptibilidade à biodegradação primária, pois apresenta 72,8 \% de EMAG insaturados, dos quais, 56,6 \% são de C18:2 enquanto que, os C16:0 e C18:0, principais produtos formados, ao final do $7^{\circ}$ dia de processo apresentaram comportamento praticamente similar e maior teor em massa $(82,00 \%)$ e $(8,70 \%)$ de seu teor de massa original $(23,5 \%)$ e $(2,97 \%)$, respectivamente. Devido à 
dificuldade de clivagem de suas ligações relacionada ao grau de insaturação que a compõem (Ferrari et al., 2005). O EMAG C18:1 que se decompôs nos 7 primeiros dias do processo, passa a ser produto da decomposição dos EMAG (7 a 14 dias), apresentando a concentração em seu máximo $(18,30 \%)$ iniciando um processo de oscilação entre ser produto de decomposição e reagente também verificado aos C16:0 e C18:0 indicando transformação entre si, como se houvesse uma ação sinérgica sobre esses até o $35^{\circ}$ dia. O B100-A respondeu a ação microbiana do consórcio cuja concentração de células viáveis foi inicialmente padronizada, indicou que é biodegradável devido à redução do $\mathrm{pH}$ e aumento elevado de acidez do meio líquido mostrado pelo $\mathrm{pH}$ : 3,57 ao $14^{\circ}$ dia atingindo ao final dos 35 dias de processo 3,14 .

\section{CONCLUSÕES}

O B100-A sofreu biodegradação primária em presença do consórcio microbiano $(P$. aeruginosa e $S$. cereviseae) verificada durante o período total de 35 dias de processo. No processo de biodegradação houve modificação significante na composição inicial dos EMAG do B100-A com formação de ácidos graxos livres de EMAG de cadeias menores que seus precursores e menos biodegradáveis devido sua instabilidade molecular relacionada a concentração de biomassa e à variação de $\mathrm{pH}$ da fase aquosa sem controle do $\mathrm{pH}$.

\section{REFERENCIAS}

CORSEUIL, H. X.; KULKAMP, M. S. Simultaneous Spills of diesel and ethanol - a controlled release experiment. IN: 7 TH INTERNATIONAL IN SITU AND ON-SITE BIOREMEDIATION SYMPOSIUM, Orlando, FL. Columbus-Richland: Battele Press, p. 1-5, 2003.

FERRARI, R.A.; OLIVEIRA,V.S.; SCABIO, A. Biodiesel de soja- taxa de conversão em ésteres etílicos, caracterização físico-química e consumo em gerador de energia. Quím. Nova, p. 19-23, 2005.

KARDEC, A. ANP discutirá adição de mais biodiesel ao diesel convencional. Disponível em: <http:// tnsustentavel.com.br/.../ANP>. Acesso em: mar. 2011.

KEMP, D. National standard for biodiesel. Setting National Fuel Quality Standards, n.6, p.1103, 2003.

MORRISON, R.; BOYD, R. Química Orgânica. Trad. de M.A. da Silva e A.J. de Gouveia. Lisboa: Fundação C. Gulbenkian,1992.

PEREIRA, L. T. C.; LEMOS, J. L. S. Degradação de hidrocarbonetos de petróleo por Aspergillus niger e Penicillium corylophilum. Disponível em: <http:// scielo.com.br〉. Acesso em: mar. 2006. 
ROCHA, M.F.A.; MARQUES, O.M. ; SARMENTO, S.M. Biodegradação Primária do Biodiesel de Algodão e da sua Mistura B5 em Meio Aquoso por Consórcio Microbiano. XVIII Simpósio Nacional de Bioprocessos. Caxias do Sul/Rio Grande do Sul, 2011.

RODRIGUES, R. B5 começa a valer em $1^{\circ}$ de janeiro de 2010. Disponível em:< http://biodieselbr.com/noticias>. Acesso em: outubro 2009.

URURAHY, A.F.P. Biorremediação de Resíduo Oleoso proveniente de Refinaria. Tese de Doutorado em Ciências. Escola de Química. Universidade Federal do Rio de Janeiro. Rio de Janeiro, 1998.

VITAL,N. Formação de emulsificantes por Candida guilliermondii T9 a partir de querosene. Dissertação de Mestrado. Escola de Química. Universidade Federal do Rio de Janeiro. Rio de Janeiro, 1992.

ZHANG,X.;PETERSON, C.L.;REECE, D.;MOLLER, G.; HAWS, R. Biodegradability of biodiesel in the aquatic environment. Trans. ASAE, p. 1423-1430,1998. 